\title{
Cesarean Section, The Reason of it Increasing Rate
}

\author{
Mauricio Besio* \\ Pontificia Universidad Católica de Chile, School of Medicine, Chile
}

*Corresponding author: Mauricio Besio, Pontificia Universidad Católica de Chile, School of Medicine, Chile, E-mail: besio@med.puc.cl

Received date: March 29, 2016; Accepted date: April 28, 2016; Published date: May 05, 2016

Copyright: ( 2016 Besio M. This is an open-access article distributed under the terms of the Creative Commons Attribution License, which permits unrestricted use, distribution, and reproduction in any medium, provided the original author and source are credited.

\section{Cesarean Section}

Medical and public opinion has been alerted by the progressive increase in the number of births that are resolved via caesarean section (C-section). There is no doubt that the improvement of technology and the advance of medical knowledge have decreased maternal risk concerning this operation, leading to a gradually reduction of the perinatal mortality. However, there seems to be consensus that the increase in the number of C-section has not kept pace with the decrease in perinatal mortality and morbidity. Similar rates of mortality and morbidity of the newborn found in centers with Csections rates ranging from $10 \%$ to almost $100 \%$ support this fact [1].

The concern seems legitimate. It is not understandable that a surgery conceived at the beginning of obstetrical art as a last resort alternative for the delivery of a newborn, has become a common, faster and maybe easier way for a human being to come to this world. Being this procedure used as or even more often than the original and natural route of birth, and without improving the conditions of the newborn. It seems necessary to answer several questions: The first is to establish what are the factors that have allowed this increase in Csection rates the second is whether this increase should really be a matter of concern. Third, which of the factors involved in the decision of performing a C-section are ethically licit, and finally what arrangements would be ethically permissible to reverse this situation.

Any attempt to resolve these issues should consider the particular and original reality of obstetrics and maternal fetal medicine: It is the only medical specialty that has to take care of two human beings, and whom should ensure equal care. Secondly, unlike in the past, today there are two different ways available to solve childbirth. Then, the obstetrician must judge in each particular case which of these two paths will be the best for the two patients.

The aim of this work is to reflect on what criteria a doctor analyzes to decide the route of delivery in a particular case, how these criteria are considered, which are taken as a priority and which are not even considered. Finally, it aims to establish some ethically licit policies to reverse this increase in $\mathrm{C}$-section rates that concerns us.

It seems imperative to present the reasons of why vaginal deliveries would be preferable. We can identify three types of reasons:

Natural: Here is invoked that pregnancy and childbirth are natural processes which develop by themselves and do not need the intervention of third parties. C-section is a surgery, a medical intervention on a process that resolves and regulates in nature without artifice.

Economics: Increased safety on C-section is not free. Childbirth resolved by this route is clearly more expensive and someone must pay for it. Therefore, from this perspective vaginal delivery is the optimum choice in terms of cost.
Medical: C-section is a medical treatment and has precise indications, and its only purpose is to ensure the health of both the mother and child. Then, it should only be performed when a vaginal delivery put them in risk.

These considerations obligate us to clarify what are the causes that might explain the increasing number of $\mathrm{C}$-sections worldwide. They can be summarized as follows:

Economics: It would be more profitable in terms of income for doctors to solve deliveries through C-section.

Convenience: C-section is faster than vaginal birth and can be scheduled during working hours. It is also more controllable, in the sense that long hours of the natural process is avoided.

Legal issues: Increasing lawsuits on bad neonatal outcome produces in doctors the feeling that it is not worth the effort to get a vaginal delivery.

From the patient: There is a fear of vaginal delivery in many women; fear of suffering a lot of pain or possible harm to their child, and also fear of genital prolapses or sexual dysfunctions. Along with this, some women would prefer an elective C-section in order to schedule it on a specific date and time.

Any of these reasons or others not mentioned above may contribute to the increasing number of C-sections. Then, it seems imperative to find the ultimate cause that achieves account and explains this reality with all its edges.

It is necessary to understand the dynamism between a subject in this case, a doctor and a medium in this case, a way of childbirth in order to obtain a goal in this case, the best result for both patients mother and child. Aristotle has pointed us that the reason for our actions is a good: "Every human activity aims at some end that we consider good" [2]. That is, we move and choose something based on some good we see in that, whatever it is an apparent or real good.

The goal of a doctor's decision is the health of the patient in this cases the mother and the child. Thus, the doctor must choose between which methods, in this case the route of delivery, will lead to get a healthy mother and a healthy newborn. A good for the doctor himself is also a goal, that is, when deciding on a route of delivery, the doctor also chooses looking for some good for him, apparent or real, more noble or less noble $[3,4]$.

Considering this, the mode that would offer the best for both patients mother and child should be priority in doctor's judgement. In the case that concerns us, the way of delivery in a particular case considered by the doctor that would achieve a healthier mother and child should be chosen. However, the doctor will not choose a determined way of delivery if he does not see any good for himself. In any medical decision the patients desire is also involved this represents autonomy, in our case the mother's choice of a particular route of 
delivery, and that is nothing more than the expression of the good that she sees for herself and/or for her child.

I present here a thesis that would explain, as a single cause, the increasing number of $\mathrm{C}$-sections and which could also explain the variability in the rate of the operation in different settings:

"The increase in the number of caesarean sections as well as their heterogeneous distribution, is basically because medical evidence that would indicate that cesarean section is more risky for the two patients than vaginal birth is insufficient to make a physician appreciate it in each particular case".

I do not intend to say that in medical literature there is not such evidence, but who has dedicated some time to look for it, may agree on the following:

It is evidence you have to look for. There are countless papers [5-7] with different methodology and some with opposite conclusions. It is difficult to obtain from them what are complications of C-section itself, or of the disease that indicated C-section. There are few studies on vaginal birth complications [8-10].

\section{Mortality and morbidity of $\mathrm{C}$-section has been gradually declining.}

While it seems certain that $\mathrm{C}$-section still poses a greater risk in the total universe of births, it does not seem to be true in those subgroups of patients with any risk factors for labor or in which the evolution of labor is no longer fluid.

In other words, and this is what I want to emphasize, the evidence from the literature and medical experience is becoming less strong in relation to the differences between routes of delivery for both physician and the patient and it only achieves to impose in the cases where the benefits of a vaginal delivery are obvious.

As the disadvantages of C-section are progressively less obvious, there are increasingly more frequent cases in which the doctor is not sure about which way to solve a birth is the best one for their patients. This is true not only during pregnancy but also during labor if added circumstances are lowering fluidity to the progress of labor. An example for this, are those deliveries in patients with cesarean scar. This situation creates a status of perplexity in the physician, when he/she has to decide which way of birth is the best for the mother and child. In these cases it seems inevitable that the consideration of the advantages for herself as well as the opinion of the mother take priority.

The increasing priority acquired by the advantage for himself and the patient's better expectations estimated for herself or her child, in cases of perplexity, will enable and explain the diversity of caesarean section rates between different doctors, different health systems and locations:

In the public system, C-sections are fewer, because the increased risk of postoperative infections makes more evident to the doctor the benefits of a vaginal birth. Besides, the doctor in public services is less pressured by personal responsibility on a patient.

In private centers, surgical complications are less common, therefore the perplexity cases are greater, the autonomy of the mother higher and the good for the doctor more oriented towards their welfare.

From the ethical point of view we can say that in the medical judgment, the choice of an action, or it's omission should consider as first priority the safety and benefit of patients. In obstetrics practice, regarding the decision of the delivery route, the physician should choose based on what type of delivery represents a better outcome for mother and newborn.

In particular cases of real clinical perplexity, if the doctor fails, based on clinical criteria, to make a judgment about the best way of delivery for his/her patient, appearing for him two ways equally valid in terms of security, it seems licit that in the choice participate the patient's preference or decision [11,12]. Letting the mother decide could be extremely controversial if the doctor is sure what type of delivery is better, in terms of health for her or her child.

Defending vaginal delivery by the reason of being a natural event would be based on full respect for biological processes as seen in nature. But natural would be then, pregnancy and childbirth as well as all the pathologies that affect them. The human being, while following his biological inclinations, is able of understand the meaning and purpose of them. His proper activity begins when he/she captivates the sense of their biological tendencies.

The purpose of the reproductive process is to ensure healthy offspring and conservation of the species.

Awareness of that sense allows us to alter these biological processes, precisely to preserve their purpose. Biological processes are explained from its aim. Man is thus capable of medicine. Caesarean section appears then as a mean to ensure that the purpose of a natural process is not distorted by an external event, being as licit as any other medical procedure.

"Economistic" kind of considerations, that defends the vaginal delivery just for being cost saving, cannot participate neither in doctor's decision over the health criteria for their patients, nor over the autonomy of the mother.

It seems also ethically permissible the existence of differences between private and institutional care, as long as it is due to different number of perplexity cases. It is perfectly possible that diverse situations or circumstances might determine, for example, a different surgical risk in different care regimes.

If the rate of C-sections needs to be decreased for legitimate reasons of resource allocation, actions must be taken to obtain a favorable disposition from mothers and their families toward vaginal birth. On the other hand, actions tending to doctors to perceive a higher good for themselves in this type of delivery should be taken as well, so that in cases of perplexity they choose the cheapest and most natural way of delivery.

\section{Conclusion}

In the controversy about the increased number of caesarean sections it is not ethically permissible that only economic or "naturalist" criteria participate in choosing the route of childbirth. Clinical judgment must always prevail in the decision: the route of delivery should be the one that ensures a better outcome for both patients, and in cases of perplexity, it would be valid to consider the mother's preference as well. Currently, with the progressive decrease in C-section complications, the proper way to decrease the frequency of this surgery should be implementing actions to strengthen the desire of the patient and physician to obtain a normal delivery. 
Citation: Besio M (2016) Cesarean Section, The Reason of it Increasing Rate. Clinics Mother Child Health 13: 237. doi:

\section{References}

1. Gibbons L, Belizan JM, Lauer JA, Betran AP, Merialdi M, et al. (2010) The Global Numbers And Costs of Additionally Needed and Unnecessary Caesarean Sections Performed Per Year: Overuse as a Barrier to Universal Coverage. World Health Report Background Paper, No 30.

2. Aristotle (350 BC) Nicomachean Ethics. Internet Classics Archive.

3. Besio M (2003) Sobre El Acto Médico. Cuadernos De Bioética 50: 25-38.

4. Besio M (2016) El Acto Médico: ¿Una Creación Original? Reflexiones Sobre Su Esencia, Surgimiento Y Riesgos A Los Que Se Expone. Acta Bioethica 16: 51-60.

5. Steer P (1998) Caesarean Section: An Evolving Procedure? Br J Obstet Gynaecol 105: 1052-1055.

6. Lilford RJ, Van Coeverden De Groot HA, Moore PJ, Binfham P (1990) The Relative Risks of Caesarean Section (Intrapartum And Elective) and Vaginal Delivery: A Detailed Analysis to Exclude the Effects of Medical Disorders and Other Acute Pre-Existing Physiological Disturbances. Br J Obstet Gynaecol 97: 883-892.
7. Sachs BP, Yeh J, Acker D, Driscoll S, Brown DA, et al. (1988) Cesarean Section Related Maternal Mortality in Massachusetts, 1954-1985. Obstet Gynecol 71: 385-388.

8. MacArthur C, Bick DE, Keighley MR (1997) Faecal Incontinence after Childbirth. Br J Obstet Gynaecol 104: 46-50.

9. Morison J, Rennie JM, Milton PJ (1995) Neonatal Respiratory Morbidity and Mode of Delivery at Term: Influence of Timing of Elective Caesarean Section. Br J Obstet Gynaecol 102: 101-106.

10. Viktrup L, Lose G, Rolff M, Barfoed K (1992) The Symptom of Stress Incontinence Caused by Pregnancy or Delivery in Primiparas. Obstet Gynecol 79: 945-949.

11. Mould Taj, Chong S, Spencer JAD, Gallivan S (1996) Women's Involvement with the Decision Preceding Their Caesarean Section and Their Degree of Satisfaction. Br J Obstet Gynaecol 103: 1074-1077.

12. Ryding EL (1993) Investigation of 33 Women Who Demanded a Cesarean Section for Personal Reasons. Acta Obstet Gynecol Scand 72: 280-285. 